\title{
Review Article \\ Spacetime Junctions and the Collapse to Black Holes in Higher Dimensions
}

\author{
Filipe C. Mena \\ Centro de Matemática, Universidade do Minho, Campus de Gualtar, 4710-057 Braga, Portugal \\ Correspondence should be addressed to Filipe C. Mena, fmena@math.uminho.pt
}

Received 30 December 2011; Revised 24 April 2012; Accepted 14 May 2012

Academic Editor: Burak Polat

Copyright (c) 2012 Filipe C. Mena. This is an open access article distributed under the Creative Commons Attribution License, which permits unrestricted use, distribution, and reproduction in any medium, provided the original work is properly cited.

We review recent results about the modelling of gravitational collapse to black holes in higher dimensions. The models are constructed through the junction of two exact solutions of the Einstein field equations: an interior collapsing fluid solution and a vacuum exterior solution. The vacuum exterior solutions are either static or containing gravitational waves. We then review the global geometrical properties of the matched solutions which, besides black holes, may include the existence of naked singularities and wormholes. In the case of radiating exteriors, we show that the data at the boundary can be chosen to be, in some sense, arbitrarily close to the data for the Schwarzschild-Tangherlini solution.

\section{Introduction}

Higher dimensional black holes have recently been the subject of an increasing number of research works (see [1] for a review) and play an important role in theoretical physics, particularly in String Theory. However, the study of the actual dynamical process of collapse resulting in black hole formation has deserved little attention from the mathematical point of view.

Mathematically, black hole formation can be analysed by constructing appropriate matched spacetimes which settle down through gravitational collapse to black hole solutions. The junction (or matching) of two spacetimes requires the equality of the respective first and second fundamental forms at some matching boundary hypersurface. These conditions amount to a set of differential equations that the spacetime metric functions need to satisfy at that matching boundary. This is often not an easy problem as the metric functions are also required to satisfy the respective Einstein field equations (EFEs) on both sides of the matching surface. In what follows, we review some results about models of black hole formation with 
emphasis on models resulting from the spacetime matching with an exterior which is either vacuum or a cosmological background.

For a zero cosmological constant $\Lambda$, there are well known spherically symmetric examples, one of the simplest being the Oppenheimer-Snyder model which results from the matching of a Friedman-Lemaître-Robertson-Walker (FLRW) dust metric to a Schwarzschild exterior. For $\Lambda \neq 0$ spherical cases, the results are less well-known: the matching of $\Lambda$-FLRW with the $\Lambda$-Schwarzschild solution (also called Kottler solution) has been investigated by Balbinot et al. [2], Nakao [3], and Markovic and Shapiro [4] and its $\Lambda$-Lemaître-TolmanBondi counterpart by Lake [5]. The matching of the dust $\Lambda$-Szekeres solution with Kottler was recently studied by Debnath et al. [6] (following a work of Bonnor [7] for $\Lambda=0$ ), while the matching of a collapsing fluid with tangential pressure and $\Lambda \neq 0$ to Kottler has been investigated by Madhav et. al. [8]. The above examples give rise to black hole solutions with $\Lambda \neq 0$ in spherical symmetry.

The collapse to nonspherical black holes has been less studied. Smith and Mann [9] have shown that one can match a collapsing $k=-1$ FLRW spacetime to an asymptotically anti-de Sitter (AdS) exterior, as a model of gravitational collapse to higher genus asymptotically AdS black holes. In a related work, Lemos [10] matched a flat FLRW metric to a radiating Vaidya exterior whose collapse results in a toroidal black hole. Subsequently, there has been considerable interest in models of toroidal and higher genus black holes (also called topological black holes), shells, and horizons, see, for example, [11-14], partly due to the existence of a "landscape" of vacua states in String theory with $\Lambda$ positive, negative, and zero (see, e.g., [15]). More recently, [16] generalised the earlier work and constructed models for the collapse of inhomogeneous and anisotropic fluids to topological asymptotically AdS black holes in planar and hyperbolic symmetry.

There is also a vast literature about the formation of black holes in cosmological models. There are, essentially, three types of models which are geometrically different, namely, models with (i) junctions with no shells where two metrics are involved, for example, swiss-cheese-type models (see, e.g., [17] and references therein); (ii) junctions with shells (these can be thin or thick), for example, models with domain walls and bubbles mentioned in the previous paragraph; (iii) no junctions, where a single metric is involved in the problem, for example, models of collapsing over-densities resulting from perturbations on cosmological backgrounds.

In the context of primordial black hole (PBH) formation, the gravitational collapse due to first-order density perturbations in FLRW models of the early universe radiative phase was first studied by Zeldovich and Novikov [18] as well as Hawking [19]. These works were then generalised $[20,21]$, in particular, to include black hole formation from the collapse of cosmic strings produced in phase transitions with symmetry breaking [22]. The collision of bubble walls formed at phase transitions was also considered as sources of PBHs in [23-25]. Different scenarios were subsequently studied and a review is in [26]. More recently, cosmological black hole formation due to QCD and electroweak phase transitions were studied by, for example, [27] following earlier works of [28] and bubble collision has been investigated in detail (for various potentials and using planar, hyperbolic, and spherical symmetry in both dS and AdS phases) by, for example, [11, 29]. Furthermore, PBH formation in cosmological models with $\Lambda \neq 0$ has very recently been analysed in [30], which contains an interesting review of numerical results up to the year 2011 (for a review on the $\Lambda=0$ case see [31]). In turn, the collapse to black holes in FLRW models have also been investigated using nonlinear scalar metric perturbations on radiative [32] and scalar field backgrounds [33], 
with those recent works establishing interesting observational bounds on the cosmological spectra nongaussianity.

In this paper, we will be mostly interested in cases which involve spacetime junctions with no shells and, in particular, we will focus on nonspherical spacetimes containing a nonzero $\Lambda$. We start by reviewing the theory of spacetime junctions and we then summarise recent results about the gravitational collapse to black holes in higher dimensions [34] which generalise the results of [16]. We start by considering a family of solutions to the $\Lambda$-vacuum Einstein equations in $n+2$ dimensions which contains black hole solutions. We find some possible interior collapsing solutions with dust as source and study the corresponding matching problem. We then analyse the global geometrical properties of the matched spacetime and find conditions for which the spacetimes have no naked singularities.

We also review the interesting case of a model of radiating gravitational collapse. In particular, we take the anisotropic Bizon-Chmaj-Schmidt (BCS) solution in $4+1$ dimensions and prove that it can be matched to some collapsing dust interiors. The BCS solution is known [35] to settle down via radiation to the Schwarzschild-Tangherlini (SchT) solution. In turn, the latter is known to be stable $[35,36]$ and we show that, for some interiors, the data at the matching surface can be chosen to be arbitrarily close to the data for a SchT exterior [34]. So, the resulting spacetime models the gravitational collapse of a fluid in five dimensions with an exterior emitting gravitational waves which settles down to the SchT solution.

\section{Geometric Theory of Spacetime Junctions in Brief}

Let $\left(M^{+}, g^{+}\right)$and $\left(M^{-}, g^{-}\right)$be two $n$-dimensional $C^{3}$ space times with oriented boundaries $\Omega^{+}$ and $\Omega^{-}$, respectively, such that $\Omega^{+}$and $\Omega^{-}$are diffeomorphic. The matched space time $(M, g)$ is the disjoint union of $M^{ \pm}$with the points in $\Omega^{ \pm}$identified such that the junction conditions are satisfied (Israel [37], Clarke and Dray [38] and Mars and Senovilla [39]). Since $\Omega^{ \pm}$are diffeomorphic, one can then view those boundaries as diffeomorphic to a 3-dimensional oriented manifold $\Omega$ which can be embedded in $M^{+}$and $M^{-}$. Let $\left\{\xi^{\alpha}\right\}$ and $\left\{x^{ \pm i}\right\}$ be coordinate systems on $\Omega$ and $M^{ \pm}$, respectively, where $\alpha, \beta=1,2, \ldots, n-1$ and $i, j=1,2, \ldots, n$. The two embeddings are given by the following $C^{3}$ maps:

$$
\begin{gathered}
\Phi^{ \pm}: \Omega \longrightarrow M^{ \pm}, \\
\xi^{\alpha} \longmapsto x^{i^{ \pm}}=\Phi^{i^{ \pm}}\left(\xi^{\alpha}\right),
\end{gathered}
$$

such that $\Omega^{ \pm} \equiv \Phi^{ \pm}(\Omega) \subset M^{ \pm}$. The diffeomorphism from $\Omega^{+}$to $\Omega^{-}$is $\Phi^{-} \circ \Phi^{+-1}$.

Given the basis $\left\{\partial /\left.\partial \xi^{\alpha}\right|_{p}\right\}$ of the tangent plane $T_{p} \Omega$ at some $p \in \Omega$, the push forwards $\left.d \Phi^{ \pm}\right|_{p} \operatorname{map}\left\{\partial /\left.\partial \xi^{\alpha}\right|_{p}\right\}$ into three linearly independent vectors at $\Phi^{ \pm}(p)$ represented by $\left.\vec{e}_{\alpha}^{ \pm}\right|_{\Phi^{ \pm}(p)}$ :

$$
d \Phi^{ \pm}\left(\left.\frac{\partial}{\partial \xi^{\alpha}}\right|_{\Omega}\right)=\left.\left.\frac{\partial \Phi^{ \pm i}}{\partial \xi^{\alpha}} \frac{\partial}{\partial x^{ \pm i}}\right|_{\Omega^{ \pm}} \equiv \vec{e}_{\alpha}^{ \pm}\right|_{\Omega^{ \pm}}=\left.e_{\alpha}^{ \pm i} \vec{e}_{\alpha}^{ \pm} \frac{\partial}{\partial x^{ \pm i}}\right|_{\Omega^{ \pm}} .
$$

On the other hand, using the pull backs $\Phi^{ \pm *}$ of the maps $\Phi^{ \pm}$, the metrics $g^{ \pm}$can be mapped to $\Omega$ given two symmetric 2-covariant tensors $\bar{g}^{+}$and $\bar{g}^{-}$whose components in the basis $\left\{d \xi^{\alpha}\right\}$ are

$$
\left.\bar{g}_{\alpha \beta}^{ \pm} \equiv e_{\alpha}^{ \pm i} e_{\beta}^{ \pm j} g_{i j}\right|_{\Omega^{ \pm}}=\left.\left(\vec{e}_{\alpha}^{ \pm} \cdot \vec{e}_{\beta}^{ \pm}\right)\right|_{\Omega^{ \pm}}
$$


The first matching conditions are given by the equality of the first fundamental forms (Israel [37])

$$
\bar{g}_{\alpha \beta}^{+}=\bar{g}_{\alpha \beta}^{-} .
$$

The bases $\left\{\left.\vec{e}_{\alpha}^{+}\right|_{p}\right\}$ and $\left\{\left.\vec{e}_{\alpha}^{-}\right|_{p}\right\}$ can then be identified,

$$
d \Phi^{+}\left(\left.\frac{\partial}{\partial \xi^{\alpha}}\right|_{\Omega}\right)=d \Phi^{-}\left(\left.\frac{\partial}{\partial \xi^{\alpha}}\right|_{\Omega}\right)
$$

as can the hypersurfaces $\Omega^{+} \equiv \Omega^{-}$, so henceforth we represent both $\Omega^{ \pm}$by $\Omega$.

We now define a 1 -form $n$, normal to the hypersurface $\Omega$, as

$$
n^{ \pm}\left(\vec{e}_{\alpha}^{ \pm}\right)=0
$$

The vectors $\left\{\vec{n}^{ \pm}, \vec{e}_{\alpha}^{ \pm}\right\}$constitute a basis on the tangent spaces to $M^{ \pm}$at $\Omega^{ \pm}$. Since the first junction condition allows the identification of $\left\{\vec{e}_{\alpha}^{+}\right\}$with $\left\{\vec{e}_{\alpha}^{-}\right\}$, we only have to ensure that both bases have the same orientation and that $n_{i}^{+} n^{+i} \stackrel{\Omega}{=} n_{i}^{-} n^{-i}$ is satisfied in order to identify the whole 4-dimensional tangent spaces of $M^{ \pm}$at $\Omega,\left\{\vec{n}^{+}, \vec{e}_{\alpha}^{+}\right\} \equiv\left\{\vec{n}^{-}, \vec{e}_{\alpha}^{-}\right\}$.

The second fundamental forms are given by

$$
K_{\alpha \beta}^{ \pm}=-n_{i}^{ \pm} e_{\alpha}^{ \pm j} \nabla_{j}^{ \pm} e_{\beta}^{ \pm i}
$$

and the second matching conditions, for nonnull surfaces, are the equality of the second fundamental forms

$$
K_{\alpha \beta}^{+}=K_{\alpha \beta}^{-}
$$

We note that these matching conditions do not depend on the choice of the normal vectors. The first matching conditions ensure the continuity of the metric across $\Omega$ while the second conditions prevent infinite jump discontinuities in the Riemann tensor so that the Einstein field equations are well defined in the distributional sense.

The theory is also fully developed for the case where the matching boundary $\Omega$ is null or changes character. In that case, the normal vectors $n_{ \pm}{ }^{\alpha}$ are substituted by the so-called rigging vectors, which are vectors nonwhere tangent to $\Omega^{ \pm}$and are used to define generalised second fundamental forms, see [39] for the details.

We are interested in the particular cases for which the matching surface inherits a certain symmetry of the two space times $\left(M^{ \pm}, g^{ \pm}\right)$. Such matching is said to preserve the symmetry. In practice one demands that the matching hypersurface is tangent to the orbits of the symmetry group to be preserved, see [40].

We note that if the exterior is a vacuum spacetime and the interior contains a fluid then the normal pressure of the fluid has to vanish at the boundary $\Omega$. This is a consequence of the fact that the matching conditions imply

$$
n_{-}^{\alpha} T_{\alpha \beta}^{-} \stackrel{\Omega}{=} n_{+}^{\alpha} T_{\alpha \beta}^{+} .
$$


We also note that if the interior contains dust then the boundary $\Omega^{-}$is ruled by geodesics, and we will explore this fact to solve the matching problem in what follows.

\section{Black Holes in Higher Dimensions}

An $(n+2)$-dimensional Lorentzian manifold $\left(M, g_{a b}\right)$ is a solution of the Einstein equations with cosmological constant $\Lambda$ and energy-momentum tensor $T_{a b}$ if its Ricci tensor $R_{a b}$ satisfies

$$
R_{a b}=\Lambda g_{a b}+\kappa\left(T_{a b}-\frac{1}{n} T g_{a b}\right)
$$

where $\kappa$ is a constant, $T=T_{a}^{a}$ and the indices $a, b=1,2, \ldots, n+2$. We will consider the family of higher-dimensional black holes given in the following proposition (see also [41, 42]).

Proposition 3.1. Let $\left(N, d \sigma^{2}\right)$ be an $n$-dimensional Riemannian Einstein manifold with Ricci scalar $n \lambda$, and let

$$
V(r)=\frac{\lambda}{n-1}-\frac{2 m}{r^{n-1}}-\frac{\Lambda r^{2}}{n+1}
$$

where $m, \Lambda$ are constants. If $J \subset \mathbb{R}$ is an open interval where $V$ is well defined and does not vanish then the $(n+2)$-dimensional Lorentzian manifold $\left(M, d s^{2}\right)$ given by $M=\mathbb{R} \times J \times N$ and

$$
d s^{2}=-V(r) d t^{2}+(V(r))^{-1} d r^{2}+r^{2} d \sigma^{2}
$$

is a solution of the vacuum Einstein equations with cosmological constant $\Lambda$.

It is easy to see that the above metric generalises the Kottler metric to arbitrary dimensions. Nevertheless, black holes with the above metric do not immediately integrate into the usual intuition of a black hole in four dimensions. For instance, since the metric on the sections of null infinity $\supset$ does not need to be a metric of constant curvature, the spacetimes are not asymptotically flat (Nor asymptotically de Sitter (dS), resp., anti de Sitter (AdS), in the case of $\Lambda>0$, resp., $\Lambda<0$.); however, by analysing the equations for null geodesics in $M$ and $N$, one can show that the spacetimes are weakly asymptotically simple [34].

Proposition 3.2. The metrics (3.3) are conformally compactifiable at infinity and are weakly asymptotically-simple.

Therefore, by comparison with the four-dimensional case [16], the following properties hold.

(i) With $\Lambda=0, \lambda>0$ and $m>0, V$ has a single zero, corresponding to an event horizon, and asymptotes to a positive constant at large $r$. This is a black-hole solution, which can be thought of as generalising the Schwarzschild metric. The (degenerate) metric on the horizon is $\mathrm{d} \sigma^{2}$, which is also the conformal metric on future null infinity $\partial^{+}$.

(ii) With $\Lambda>0, m>0$ and large enough positive $\lambda, V(r)$ is positive between two zeroes, corresponding to a black-hole event horizon and a cosmological event horizon. The solution generalises the asymptotically dS Kottler solution. 
(iii) With $\Lambda<0$ and $m>0, V$ again has a single zero, corresponding to an event horizon, and the solution generalises the asymptotically AdS Kottler solution.

(iv) The solutions in the previous class with $\lambda \leq 0$ may have no global symmetries except the staticity Killing vector. This is because compact, negative scalar curvature Einstein manifolds have no global symmetries, nor does, for example, the Ricci-flat metric on $K 3$ (an example with $\lambda=0$ and $n=4$ ).

\subsection{Generalised Friedman-Lemaître-Robertson-Walker Interiors}

We will fill in the solutions of the previous section with (interior) collapsing dust solutions, so that the resulting global spacetime is a model of gravitational collapse with a black hole as the end state. In order to do that, we take the following classes of generalised FLRW spacetimes.

Proposition 3.3. The $(n+2)$-dimensional Lorentzian metric,

$$
d s^{2}=-d \tau^{2}+R^{2}(\tau)\left(d \rho^{2}+f^{2}(\rho) d \sigma^{2}\right)
$$

is a solution of the Einstein equations with cosmological constant $\Lambda$ and energy-momentum tensor $T_{a b}=\mu u_{a} u_{b}$, corresponding to a dust fluid with density $\mu$ and velocity $u_{a} d x^{a}=d \tau$, if and only if $R(\tau)$ and $\mu(\tau)$ satisfy the conservation equation:

$$
\mu R^{n+1}=\mu_{0}
$$

for constant $\mu_{0}$, and the Friedman-like equation:

$$
\frac{\dot{R}^{2}}{R^{2}}+\frac{k}{n R^{2}}=\frac{2 \kappa \mu}{n(n+1)}+\frac{\Lambda}{n+1}
$$

We consider the matching of an interior metric (3.4) to a static exterior (3.3) at a hypersurface $\Omega$ ruled by radial time-like geodesics in (3.3), that is, a surface of constant $\rho$ (say $\rho=\rho_{0}$ ) in (3.4). We then solve the matching conditions (2.5) and (2.9) together with the EFEs (3.6) to get the following result [34].

Theorem 3.4. The metric (3.3) can be matched to the FLRW-like metric (3.4) at $\rho=\rho_{0}$ provided that $f^{\prime}\left(\rho_{0}\right)>0$ and $m=\kappa \mu_{0} f\left(\rho_{0}\right)^{n+1} / n(n+1)$.

The matching boundary is comoving with the collapsing fluid whose dynamics is given by (3.6). We call the matched spacetime of the previous theorem FLRW-Kottler spacetime. We now summarize some properties of the FLRW-Kottler spacetime in the three cases $\Lambda=0, \Lambda>0$, and $\Lambda<0$ : when the Einstein manifold $N$ is not cobordant to a point (e.g., $\mathbb{C} P^{2}$ ) the solutions we find cannot have a regular origin, though they can be regular with spacetime wormholes or a "cusp" at the origin. When there is a singularity at the origin, it may or may not be visible from infinity. If the singularity is visible it is called naked singularity as it is not hidden by an horizon. The next three propositions exploit these aspects, and Figures 1, 2, and 3 show Penrose diagrams which represent the global structure of the matched spacetimes in the cases $\Lambda=0, \Lambda<0$, and $\Lambda>0$ [34]. In particular, by comparing the conformal lifetime 


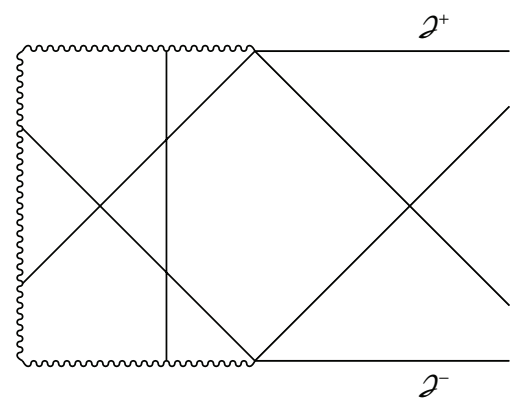

(a)

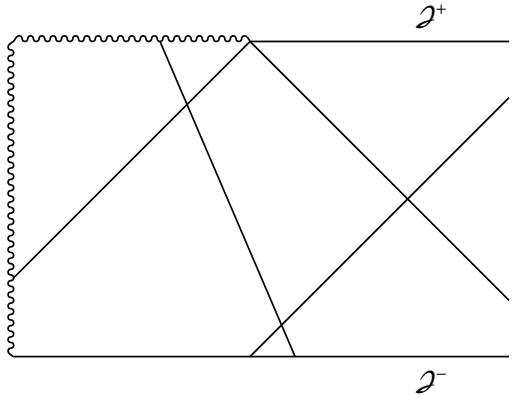

(b)

Figure 1: Penrose diagram for $\Lambda>0$ with the FLRW universe (a) recollapsing; (b) nonrecollapsing, showing the matching surfaces and the horizons.

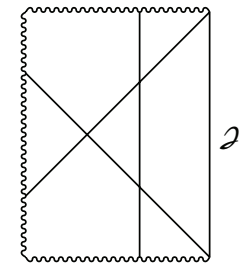

(a)

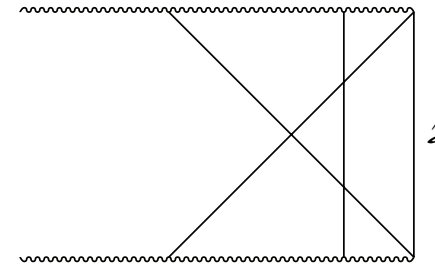

(b)

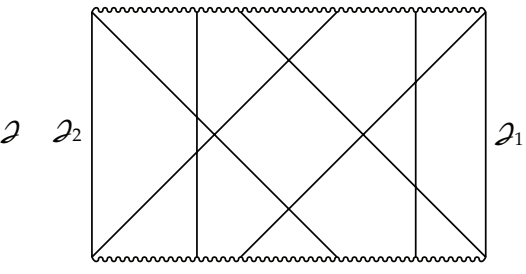

(c)

Figure 2: Penrose diagram for $\Lambda<0$ and (a) $\lambda>0$; (b) $\lambda=0$; (c) $\lambda<0$, showing the matching surfaces and the horizons.

of the FLRW universe with the supremum of the possible values of $\rho_{0}$ we show [34] the following.

Proposition 3.5. If $\Lambda=0$ (hence $\lambda>0$ ) and $\left(N, d \sigma^{2}\right)$ is not an $n$-sphere then the locally naked singularity of the FLRW-Kottler spacetime at $\rho=0$ is always visible from future null infinity $\supset^{+}$for $k \leq 0$, but can be hidden if $k>0$ and $n \geq 4$ (space-time dimension $n+2 \geq 6$ ).

Interestingly, [43] investigated higher dimensional dust collapsing spacetimes with $\Lambda=0$ and also found that for dimension $d=n+2 \geq 6$ the final central singularity is always hidden by an horizon, while for $d<6$ naked singularities may appear and thus the cosmic censorship conjecture is violated. By construction, their collapsing interiors are spherical, while here we allow interior spacetimes with other topologies.

Proposition 3.6. If $\Lambda>0$ (hence $\lambda>0$ ) and $\left(N, d \sigma^{2}\right)$ is not an $n$-sphere then the locally naked singularity of the FLRW-Kottler spacetime at $\rho=0$ can be always hidden except if the FLRW universe is recollapsing (hence $k>0$ ) and $n<4$.

The case $\Lambda<0$ has wormhole solutions that may have causal curves which cross from one 2 to the other and may violate causality. The next proposition, in particular, states conditions under which this can be avoided (see also a result of Galloway [44]). Those conditions are obtained by showing that the future (resp., past) horizons hit the matching 


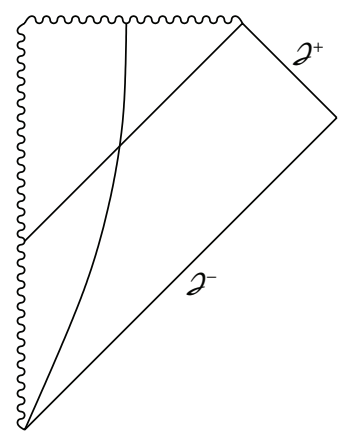

(a)

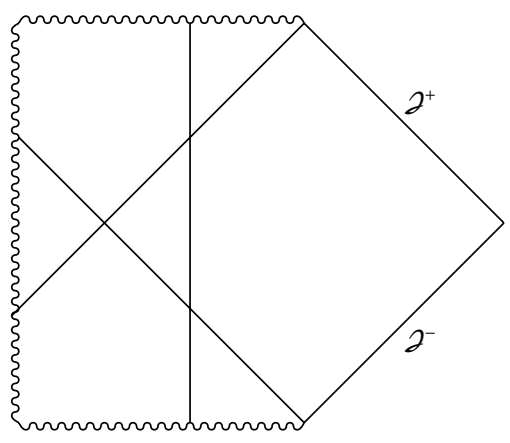

(b)

Figure 3: Penrose diagram for $\Lambda=0$ and (a) $k \leq 0$; (b) $k>0$, showing the matching surfaces and the horizons.

surfaces at marginally outer trapped (resp., antitrapped) surfaces which are spacelike and, in turn, that these two curves touch at $\dot{R}=\rho=0$.

Proposition 3.7. For $\Lambda<0$ the FLRW-Kottler spacetime satisfies the following.

(1) If $\lambda>0$ and $\left(N, d \sigma^{2}\right)$ is not an $n$-sphere then the locally naked singularity of the FLRWKottler spacetime at $\rho=0$ can always be hidden.

(2) If $\lambda=0$ then the cusp singularity is not locally naked.

(3) If $\lambda<0$ then no causal curve can cross the wormhole from one $\supset$ to the other.

\subsection{Generalised Lemaître-Tolman-Bondi Interiors}

We consider now higher dimensional versions of the inhomogeneous Lemaittre-TolmanBondi (LTB) solutions, generalising those of [45].

Proposition 3.8. The $(n+2)$-dimensional Lorentzian metric,

$$
d s^{2}=-d \tau^{2}+A(\tau, \rho)^{2} d \rho^{2}+B(\tau, \rho)^{2} d \sigma^{2},
$$

is a solution of the Einstein equations with cosmological constant $\Lambda$ and energy-momentum tensor $T_{a b}=\mu u_{a} u_{b}$, corresponding to a dust fluid with density $\mu$ and velocity $u_{a} d x^{a}=d \tau$, if and only if $A(\tau, \rho), B(\tau, \rho)$ and $\mu(\tau, \rho)$ satisfy

$$
\begin{gathered}
A=B^{\prime}(1+w(\rho)), \\
\mu A B^{n}=M^{\prime}(\rho)(1+w(\rho)),
\end{gathered}
$$

for some functions $w(\rho)$ and $M(\rho)$, and

$$
\dot{B}^{2} B^{n-1}+\left(\frac{\lambda}{n-1}-\frac{1}{(1+w(\rho))^{2}}-\frac{\Lambda}{n+1} B^{2}\right) B^{n-1}=\frac{2 \kappa M(\rho)}{n},
$$

(where dot and prime denote differentiation with respect to $\tau$ and $\rho$ ). 
This metric has three free functions of $\rho$, namely, $w(\rho), M(\rho)$, and $B(0, \rho)$, one of which can be removed by coordinate freedom. We seek to match an interior represented by the metric (3.7) to a static exterior represented by the metric (3.3) at a surface $\Omega$ ruled by radial time-like geodesics in (3.3), that is, a surface of constant $\rho$ (say $\rho=\rho_{0}$ ) in (3.7). The solutions to the matching conditions (2.5) and (2.9) allow us to prove [34].

Theorem 3.9. The metric (3.3) can be matched to the LTB-like metric (3.7) at $\rho=\rho_{0}$ provided that $1+w\left(\rho_{0}\right)>0$ and $m=(\kappa / n) M\left(\rho_{0}\right)$.

Note that the matching boundary is again comoving with the collapsing inhomogeneous fluid and its dynamics is now given by (3.9). We will use the term LTBKottler space-times for these matched solutions. The global properties of the LTB-Kottler spacetime obtained in Theorem 3.9 are much more diverse than what we find in the FLRWKottler spacetimes. For instance, one can easily find examples of black hole formation with wormholes inside the matter with positive $\lambda$ and $\Lambda=0$, see [34] (similar results in 4 dimensions are in [46]).

\section{Collapse with Radiating Exteriors}

In this section, we consider models of gravitational collapse with a gravitational wave exterior, so that the exterior metrics will be time-dependent generalisations of (3.3). This is a specially interesting problem for observational and experimental purposes [36]. We take the Bizon-Chmaj-Schmidt (BCS) metric in $(4+1)$ dimensions [36], although similar ansetz can be made in other dimensions (see [47]). We then consider three different interiors with this exterior, which are anisotropic generalisations of FLRW models [34]. Consider the metric:

$$
d s^{2+}=-A e^{-2 \delta} d t^{2}+A^{-1} d r^{2}+\frac{r^{2}}{4} e^{2 B}\left(\sigma_{1}^{2}+\sigma_{2}^{2}\right)+\frac{r^{2}}{4} e^{-4 B} \sigma_{3}^{2}
$$

where $A, \delta$, and $B$ are functions of $t$ and $r$. The one forms $\sigma_{i}$ are left invariant for the standard Lie group structure on $S^{3}$ and can be taken as

$$
\begin{gathered}
\sigma_{1}=\cos \psi d \theta+\sin \theta \sin \psi d \phi, \\
\sigma_{2}=\sin \psi d \theta-\sin \theta \cos \psi d \phi, \\
\sigma_{3}=d \psi+\cos \theta d \phi,
\end{gathered}
$$

where $\theta, \psi, \phi$ are Euler angles on $S^{3}$ with $0<\theta<\pi, 0<\phi<2 \pi$, and $0<\psi<4 \pi$. The spacetime with $B \neq 0$ is interpreted as containing pure gravitational waves with radial symmetry [36] and the Schwarzschild-Tangherlini (SchT) limit is obtained for $B=0$. There is a residual coordinate freedom $t \rightarrow \widehat{t}=f(t) ; \delta \rightarrow \widehat{\delta}=\delta+\log \dot{f}$ in (4.1), which one can use to set $\delta$ arbitrarily along a timelike curve. The $(4+1)$ dimensional vacuum EFEs give [36]

$$
\begin{gathered}
\partial_{r} A=-\frac{2 A}{r}+\frac{1}{3 r}\left(8 e^{-2 B}-2 e^{-8 B}\right)-2 r\left(e^{2 \delta} A^{-1}\left(\partial_{t} B\right)^{2}+A\left(\partial_{r} B\right)^{2}\right), \\
\partial_{t} A=-4 r A\left(\partial_{t} B\right)\left(\partial_{r} B\right),
\end{gathered}
$$




$$
\partial_{r} \delta=-2 r\left(e^{2 \delta} A^{-2}\left(\partial_{t} B\right)^{2}+\left(\partial_{r} B\right)^{2}\right),
$$

together with the quasi-linear wave equation for $B$

$$
\partial_{t}\left(e^{\delta} A^{-1} r^{3}\left(\partial_{t} B\right)\right)-\partial_{r}\left(e^{-\delta} A r^{3}\left(\partial_{r} B\right)\right)+\frac{4}{3} e^{-\delta} r\left(e^{-2 B}-e^{-8 B}\right)=0
$$

In [36], the authors solve this system numerically by giving $B$ and $\partial_{t} B$ at $t=0$ with $A(0,0)=$ $0=\delta(t, 0)$. Within the BCS class, they provide numerical evidence that linear perturbations of SchT decay in time so that SchT is linearly stable, in this sense. The nonlinear stability of SchT within the BCS class was later established in [35].

We will give data $A, B$ and the normal derivative $\nabla_{n} B$ at the timelike boundary

$\Omega$ of the collapsing interior with the gauge choice $\delta \stackrel{\Omega}{=} 0$. Uniqueness and local existence follow as standard. From [35], one knows that if data close to that for SchT is given on an asymptotically flat hypersurface then the solution will exist forever and stay close to the SchT solution. As we will see, in some of our cases, data on $\Omega$ can be chosen to be arbitrarily close to data for SchT.

\subsection{Generalised FLRW Interiors}

As interior metrics, we will consider three classes of FLRW-like solutions based on Riemannian Bianchi-IX spatial metrics which are, respectively, the Eguchi-Hanson metric (with $R_{i j}=0$ ), the $k$-Eguchi-Hanson metric (with $R_{i j}=k g_{i j}$ excluding the case $k=0$ ), and the $k$-Taub-NUT metric (with $R_{i j}=k g_{i j}$, including, $k=0$ as a particular case).

We summarize now our main result and leave the details of the proof to the next three sections (see also [34]).

Theorem 4.1. In each case, the interior metric gives consistent data for the metric (4.1) at a comoving time-like hypersurface. Local existence of the radiating exterior in the neighbourhood of the matching surface is then guaranteed. In the case of Eguchi-Hanson and $k$-Taub-NUT with $k<0$, the data can be chosen to be close to the data for the Schwarzschild-Tangherlini solution.

\subsubsection{The Eguchi-Hanson Metric}

Eguchi and Hanson found a class of self-dual solutions to the Euclidean Einstein equations with metric given by [48]

$$
h_{\mathrm{EH}}=\left(1-\frac{a^{4}}{\rho^{4}}\right)^{-1} d \rho^{2}+\frac{\rho^{2}}{4}\left(\sigma_{1}^{2}+\sigma_{2}^{2}\right)+\frac{\rho^{2}}{4}\left(1-\frac{a^{4}}{\rho^{4}}\right) \sigma_{3}^{2},
$$

with $\sigma_{i}$ given by (4.2) and $a$ is a real constant. The generalised FLRW metric built on this Riemannian metric is

$$
d s^{2-}=-d \tau^{2}+R^{2}(\tau) h_{\mathrm{EH}},
$$


with the EFEs for a dust source reducing to

$$
\mu R^{4}=\mu_{0}, \quad \dot{R}^{2}=\frac{\kappa \mu_{0}}{6 R^{2}} .
$$

We match at $\rho=\rho_{0}$ so that $\Omega^{-}$is parameterised by $\Omega^{-}=\left\{\tau, \rho=\rho_{0}\right\}$. The equality of the first and second fundamental forms at $\Omega$ then gives

$$
r \stackrel{\Omega}{=} R \rho e^{-B}, \quad e^{-6 B} \stackrel{\Omega}{=} 1-\frac{a^{4}}{\rho^{4}}, \quad \nabla_{n} B \stackrel{\Omega}{=}-\frac{2 a^{4}}{3 R \rho^{5}}\left(1-\frac{a^{4}}{\rho^{4}}\right)^{-1 / 2}, \quad A e^{-\delta} \dot{t} \stackrel{\Omega}{=} e^{2 B}\left(1-\frac{a^{4}}{3 \rho^{4}}\right),
$$

from which we calculate $A, \partial_{t} B$, and $\partial_{r} B$ on $\Omega$ in terms of quantities from the interior. At this point, we have $B, \nabla_{n} B$, and $A$ on $\Omega$, and we use the gauge freedom to set $\delta=0$ on $\Omega$. By (4.10), we have

$$
B \stackrel{\Omega}{=} O\left(\frac{a^{4}}{\rho^{4}}\right), \quad \nabla_{n} B \stackrel{\Omega}{=}(\rho R)^{-1} O\left(\frac{a^{4}}{\rho^{4}}\right),
$$

and, to say that the data is close to SchT data, we want these to be small. The first term is small if $\rho \gg a$. The second term will increase without bound as $R$ decreases to zero. If we restrict $R$ by its value when a marginally outer trapped surface forms on $\Omega$ then, from the Friedman equation and with $\rho \gg a$, this happens when $R^{2} \rho^{2} \sim \kappa \mu_{0} \rho^{4}$ so that we control $\nabla_{n} B$ on $\Omega$ by controlling $\mu_{0}$. So, by choice of the location of $\Omega$, at $\rho=\rho_{0}$, and choice of $\mu_{0}$, we can choose data close to SchT.

\subsubsection{The k-Eguchi-Hanson Metric}

The $k$-Eguchi-Hanson metric is given by [49]

$$
h_{k \mathrm{EH}}=\Delta^{-1} d \rho^{2}+\frac{\rho^{2}}{4}\left(\sigma_{1}^{2}+\sigma_{2}^{2}\right)+\frac{\rho^{2}}{4} \Delta \sigma_{3}^{2}
$$

where $\Delta=1-a^{4} / \rho^{4}-k \rho^{2} / 6$. This metric is complete for $k<0$ and $a^{4}=4(p-2)^{2}(p+1) / 3 k^{2}$, $\rho>(-2(p-2) / k)^{1 / 2}$, where $p \geq 3$ in an integer. Since $k$ is related to $a$ for a complete solution, we cannot obtain the previous case from this case by taking $k \rightarrow 0$. However, the matching formulae do formally allow this limit. The generalised FLRW metric built on this metric is $d s^{2-}=-d \tau^{2}+R^{2}(\tau) h_{k \mathrm{EH}}$ and the respective EFEs for a dust source reduce to

$$
\mu R^{4}=\mu_{0}, \quad \dot{R}^{2}+\frac{k}{3}=\frac{\kappa \mu_{0}}{6 R^{2}} .
$$

The matching conditions on $\Omega$ give

$$
r \stackrel{\Omega}{=} R \rho e^{-B}, \quad e^{-6 B} \stackrel{\Omega}{=} \Delta, \quad \nabla_{n} B \stackrel{\Omega}{=}-\frac{\Delta^{-1 / 2}}{3 \rho R}\left(\frac{2 a^{4}}{\rho^{4}}-\frac{k \rho^{2}}{6}\right), \quad A e^{-\delta} \dot{t} \stackrel{\Omega}{=} e^{2 B}\left(1-\frac{a^{4}}{3 \rho^{4}}-\frac{2 k \rho^{2}}{9}\right) .
$$


We can calculate $A$ in terms of interior data, as before, and check that this is consistent with the EFEs (4.3) and (4.4). However, in this case, it is not clear that we may choose data close to SchT data as the normal derivative of $B$ is

$$
\nabla_{n} B \stackrel{\Omega}{=} \frac{k \rho}{6 R}
$$

and for this to be small, we would require $R$ to be large on $\Omega$ outside the marginally trapped surface. It is hard to see how to arrange this and so although the solution in the exterior exists locally, we do not have a good reason to think that it will settle down to SchT.

\subsection{3. $k$-Taub-NUT}

We consider the Riemannian Taub-NUT metric with a cosmological constant $(k$ rather than $\Lambda$ with our conventions) $[50,51]$

$$
h_{\mathrm{TN}}=\frac{1}{4} \Sigma^{-1} d \rho^{2}+\frac{1}{4}\left(\rho^{2}-L^{2}\right)\left(\sigma_{1}^{2}+\sigma_{2}^{2}\right)+L^{2} \Sigma \sigma_{3}^{2}
$$

where $\Sigma=(\rho-L)(1-(k / 12)(\rho-L)(\rho+3 L)) /(\rho+L)$, and use it to construct the $4+1$ interior:

$$
d s^{2-}=-d \tau^{2}+R^{2}(\tau) h_{\mathrm{TN}}
$$

The EFEs for a dust source are again (4.13). From matching the first fundamental forms we get

$$
r \stackrel{\Omega}{=} R\left(\rho^{2}-L^{2}\right)^{1 / 2} e^{-B}, \quad e^{-6 B} \stackrel{\Omega}{=} \frac{4 L^{2} \Sigma}{\rho^{2}-L^{2}},
$$

and the second matching conditions read

$$
A e^{-\delta} \dot{t} \stackrel{\Omega}{=} \frac{4 R}{3 r} \Sigma^{1 / 2} e^{4 B}\left(\frac{2 L^{2}(2 \rho+L) \Sigma}{\rho^{2}-L^{2}}-\frac{k}{6} L^{2}(\rho-L)\right), \quad \nabla_{n} B \stackrel{\Omega}{=} \frac{1}{3 R}\left(\frac{2 \Sigma^{1 / 2}}{\rho+L}+\frac{k(\rho-L)}{6 \Sigma^{1 / 2}}\right) .
$$

As before, the expression for $A$ on $\Omega$ is consistent with $\dot{A}$ calculated from (4.3) and (4.4). Now note that if $k L^{2}=-3$ then the metric (4.16) is precisely the 4-dimensional hyperbolic metric. In this case, $B$ and $\nabla_{n} B$ vanish on $\Omega$ whatever is the value of $\rho_{0}$, so that the exterior metric is precisely SchT: this is a case from the previous section as the interior is now a standard FLRW cosmology. Consequently, if we take $k L^{2}$ close to -3 we get data close to SchT data. To see this, set $k L^{2}=-3(1+\varepsilon)$. Then $\Sigma=\left(\left(\rho^{2}-L^{2}\right) / 4 L^{2}\right)(1+O(\varepsilon))$, so that

$$
e^{-6 B} \stackrel{\Omega}{=} 1+O(\varepsilon), \quad \nabla_{n} B \stackrel{\Omega}{=} \frac{1}{L R} O(\varepsilon)
$$

and the data $\left(B, \nabla_{n} B\right)$ can be chosen as small as desired by choosing large $\rho_{0}$ and small $\varepsilon$. 


\section{Acknowledgments}

The work summarised in this paper was done in collaboration with José Natário and Paul Tod. The author is supported by FCT Projects PTDC/MAT/108921/2008, CERN/FP/ 116377/2010, and Est-C/MAT/UI0013/2011 and by CMAT, Universidade do Minho, through FCT plurianual FEDER Funds "Programa Operacional Factores de Competitividade COMPETE."

\section{References}

[1] R. Emparan and H. S. Reall, "Black holes in higher dimensions," Living Reviews in Relativity, vol. 11, p. 6, 2008.

[2] R. Balbinot, R. Bergamini, and A. Comastri, "Solution of the Einstein-Straus problem with a $\Lambda$ term," Physical Review D, vol. 38, no. 8, pp. 2415-2418, 1988.

[3] K. Nakao, "The Oppenheimer-Snyder space-time with a cosmological constant," General Relativity and Gravitation, vol. 24, no. 10, pp. 1069-1081, 1992.

[4] D. Markovic and S. L. Shapiro, "Gravitational collapse with a cosmological constant," Physical Review D, vol. 61, no. 8, 084029, pp. 1-7, 2000.

[5] K. Lake, "Gravitational collapse of dust with a cosmological constant," Physical Review D, vol. 62, no. 2, 027301, 2000.

[6] U. Debnath, S. Nath, and S. Chakraborty, "Quasi-spherical collapse with cosmological constant," Monthly Notices of the Royal Astronomical Society, vol. 369, no. 4, pp. 1961-1964, 2006.

[7] W. B. Bonnor, "Non-radiative solutions of Einstein's equations for dust," Communications in Mathematical Physics, vol. 51, no. 3, pp. 191-199, 1976.

[8] T. A. Madhav, R. Goswami, and P. S. Joshi, "Gravitational collapse in asymptotically anti-de Sitter or de Sitter backgrounds," Physical Review D, vol. 72, no. 8, 084029, 2005.

[9] W. L. Smith and R. B. Mann, "Formation of topological black holes from gravitational collapse," Physical Review D, vol. 56, no. 8, pp. 4942-4947, 1997.

[10] J. P. S. Lemos, "Gravitational collapse to toroidal, cylindrical, and planar black holes," Physical Review D, vol. 57, no. 8, pp. 4600-4605, 1998.

[11] B. Freivogel, G. T. Horowitz, and S. Shenker, "Colliding with a crunching bubble," Journal of High Energy Physics, no. 5, p. 090, 2007.

[12] Yumei Wu, M. F A Da Silva, N. O. Santos, and Anzhong Wang, "Topological charged black holes in high dimensional spacetimes and their formation from gravitational collapse of a type II fluid," Physical Review D, vol. 68, no. 8, 084012, 2003.

[13] D. Ida, T. Shiromizu, and H. Ochiai, "Semiclassical instability of the brane-world: Randall-Sundrum bubbles," Physical Review D, vol. 65, no. 2, 023504, 2002.

[14] D. Birmingham and M. Rinaldi, "Bubbles in anti-de Sitter space," Physics Letters B, vol. 544, no. 3-4, pp. 316-320, 2002.

[15] M. R. Douglas and S. Kachru, "Flux compactification," Reviews of Modern Physics, vol. 79, no. 2, pp. 733-796, 2007.

[16] F. C. Mena, J. Natário, and P. Tod, “Gravitational collapse to toroidal and higher genus asymptotically AdS black holes," Advances in Theoretical and Mathematical Physics, vol. 12, no. 5, pp. 1163-1181, 2008.

[17] A. Krasinski, Inhomogeneous Cosmological Models, Cambridge University Press, 1997.

[18] Ya. B. Zeldovich and I. D. Novikov, "The hypothesis of cores retarded during expansion and the hot cosmological model," Soviet Astronomy, vol. 10, p. 602, 1967.

[19] S. Hawking, "Gravitationally collapsed objects of very low mass," Monthly Notices of the Royal Astronomical Society, vol. 152, p. 75, 1971.

[20] J. B. Carr and S. W. Hawking, "Black holes in the early universe," Monthly Notices of the Royal Astronomical Society, vol. 168, pp. 399-416, 1974.

[21] A. G. Polnarev and M. Yu. Khlopov, "Cosmology, primordial black holes, and supermassive particles," Soviet Physics Uspekhi, vol. 145, pp. 213-232, 1985.

[22] S. W. Hawking, "Black holes from cosmic strings," Physics Letters B, vol. 231, no. 3, pp. 237-239, 1989.

[23] S. W. Hawking, I. G. Moss, and J. M. Stewart, "Bubble collisions in the very early universe," Physical Review D, vol. 26, no. 10, pp. 2681-2693, 1982. 
[24] I. G. Moss, "Singularity formation from colliding bubbles," Physical Review D, vol. 50, no. 2, pp. 676681, 1994.

[25] M. Yu. Khlopov, R. V. Konoplich, S. G. Rubin, and A. S. Sakharov, "First order phase transitions as a source of black holes in the early Universe," Gravitation \& Cosmology, vol. 23, pp. 153-156, 2000.

[26] B. J. Carr, "Primordial black holes as a probe of cosmology and high energy physics," in Quantum Gravity, vol. 631 of Lecture Notes in Physics, pp. 301-321, 2003.

[27] J. I. Kapusta and T. Springer, "Potentials for soft-wall AdS/QCD," Physical Review D, vol. 81, no. 8, 086009, 2010.

[28] K. Jedamzik, "Primordial black hole formation during the QCD epoch," Physical Review D, vol. 55, no. 10, pp. R5871-R5875, 1997.

[29] D. Hwang, B. Lee, W. Lee, and D. Yeom, “Bubble collision with gravitation," 2012, http://arxiv.org/ abs/1201.6109.

[30] I. Musco, "Critical collapse and black hole formation within an expanding perfect fluid," 2011, http://arxiv.org/abs/1106.5287.

[31] B. J. Carr, T. Harada, and H. Maeda, "Can a primordial black hole or wormhole grow as fast as the universe?" Classical and Quantum Gravity, vol. 27, no. 18, p. 183101, 2010.

[32] M. Shibata and M. Sasaki, "Black hole formation in the Friedmann universe: formulation and computation in numerical relativity," Physical Review D, vol. 60, no. 8, 084002, 1999.

[33] J. C. Hidalgo, Primordial Black Holes in non-linear perturbation theory [Ph.D. thesis], Queen Mary, University of London, 2009, http:/ / arxiv.org/abs/0910.1876.

[34] F. C. Mena, J. Natário, and P. Tod, “Formation of higher-dimensional topological black holes," Annales Henri Poincaré, vol. 10, no. 7, pp. 1359-1376, 2010.

[35] M. Dafermos and G. Holzegel, "On the nonlinear stability of higher dimensional triaxial Bianchi-IX black holes," Advances in Theoretical and Mathematical Physics, vol. 10, no. 4, pp. 503-523, 2006.

[36] P. Bizon, T. Chmaj, and B. G. Schmidt, "Critical behavior in vacuum gravitational collapse in $4+1$ dimensions," Physical Review Letters, vol. 95, no. 7, p. 071102, 2005.

[37] W. Israel, "Singular hypersurfaces and thin shells in general relativity," Il Nuovo Cimento B, vol. 44, no. 1 , pp. 1-14, 1966.

[38] C. J. S. Clarke and T. Dray, "Junction conditions for null hypersurfaces," Classical and Quantum Gravity, vol. 4, no. 2, pp. 265-275, 1987.

[39] M. Mars and J. M. M. Senovilla, "Geometry of general hypersurfaces in spacetime: junction conditions," Classical and Quantum Gravity, vol. 10, no. 9, pp. 1865-1897, 1993.

[40] R. Vera, "Symmetry-preserving matchings," Classical and Quantum Gravity, vol. 19, no. 20, pp. 52495264, 2002.

[41] G. Gibbons and S. A. Hartnoll, "Gravitational instability in higher dimensions," Physical Review D, vol. 66, no. 6, 064024, 2002.

[42] G. W. Gibbons, D. Ida, and T. Shiromizu, “Uniqueness and non-uniqueness of static vacuum black holes in higher dimensions," Progress of Theoretical Physics. Supplement, no. 148, pp. 284-290, 2002.

[43] R. Goswami and P. S. Joshi, "Cosmic censorship in higher dimensions," Physical Review D, vol. 69, no. 10, 104002, 2004.

[44] G. J. Galloway, "A "finite infinity" version of topological censorship," Classical and Quantum Gravity, vol. 13, no. 6, pp. 1471-1478, 1996.

[45] S. G. Ghosh and A. Beesham, "Higher dimensional inhomogeneous dust collapse and cosmic censorship," Physical Review D, vol. 64, no. 12, 124005, 2001.

[46] C. Hellaby, "A Kruskal-like model with finite density," Classical and Quantum Gravity, vol. 4, no. 3, pp. 635-650, 1987.

[47] P. Bizoń, T. Chmaj, A. Rostworowski, B. G. Schmidt, and Z. Tabor, "Vacuum gravitational collapse in nine dimensions," Physical Review D, vol. 72, no. 12, 121502, 2005.

[48] T. Eguchi and A. J. Hanson, "Asymptotically flat self-dual solutions to euclidean gravity," Physics Letters B, vol. 74, no. 3, pp. 249-251, 1978.

[49] H. Pedersen, "Eguchi-Hanson metrics with cosmological constant," Classical and Quantum Gravity, vol. 2, pp. 579-587, 1985.

[50] H. Boutaleb-Joutei, "The general Taub-NUT-de Sitter metric as a self-dual Yang-Mills solution of gravity," Physics Letters B, vol. 90, no. 1-2, pp. 181-184, 1980.

[51] M. M. Akbar, "Classical boundary-value problem in Riemannian quantum gravity and Taub-boltanti-de Sitter geometries," Nuclear Physics B, vol. 663, no. 1-2, pp. 215-230, 2003. 


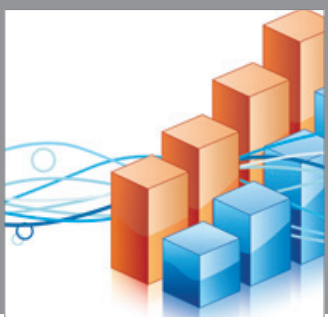

Advances in

Operations Research

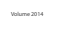

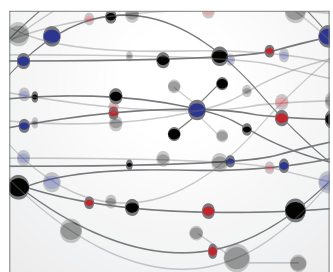

\section{The Scientific} World Journal
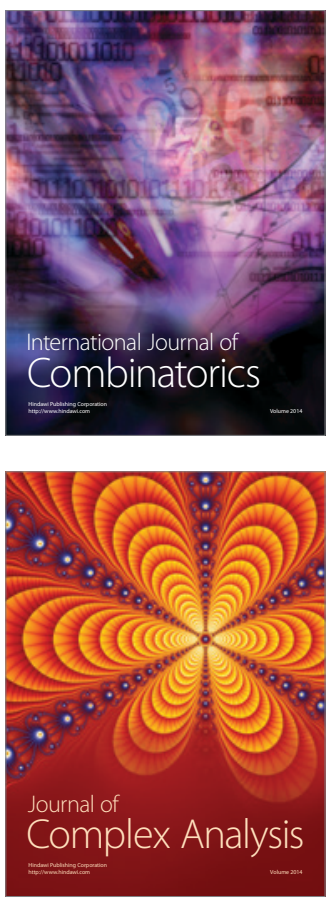

International Journal of

Mathematics and

Mathematical

Sciences
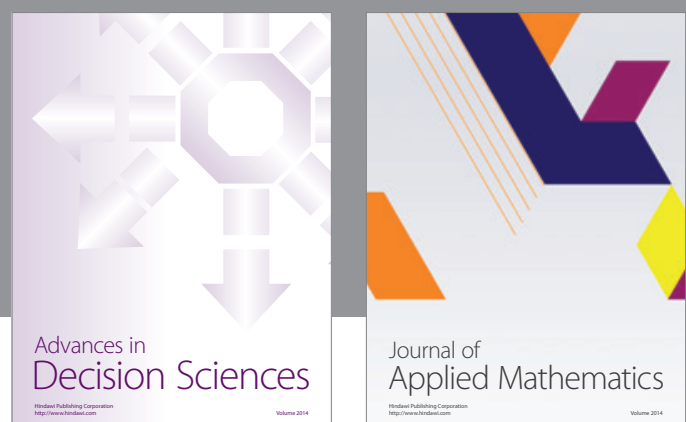

Journal of

Applied Mathematics
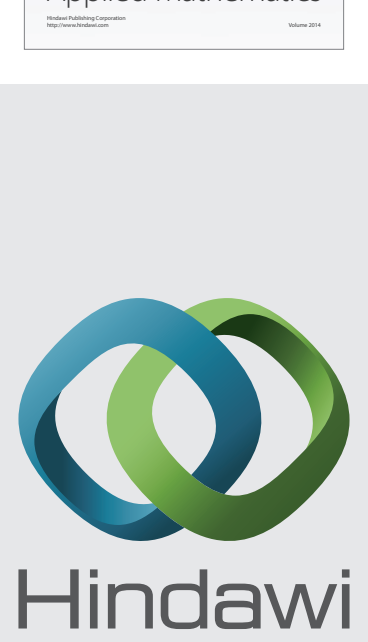

Submit your manuscripts at http://www.hindawi.com
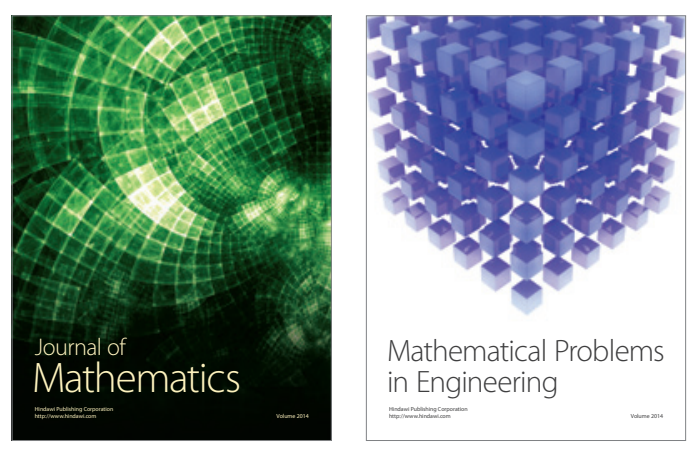

Mathematical Problems in Engineering
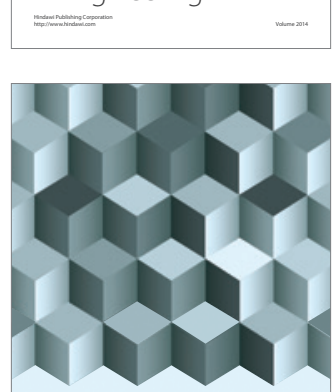

Journal of

Function Spaces
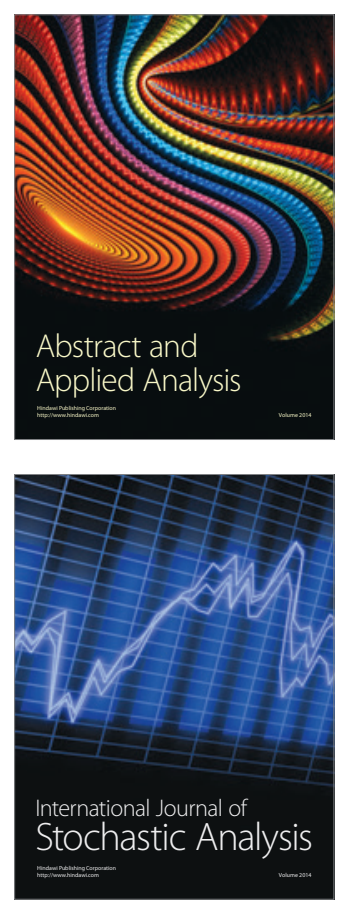

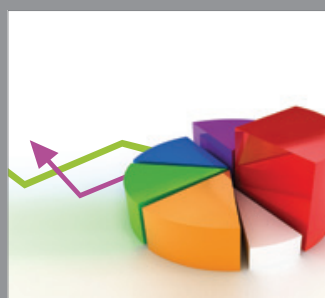

ournal of

Probability and Statistics

Promensencen
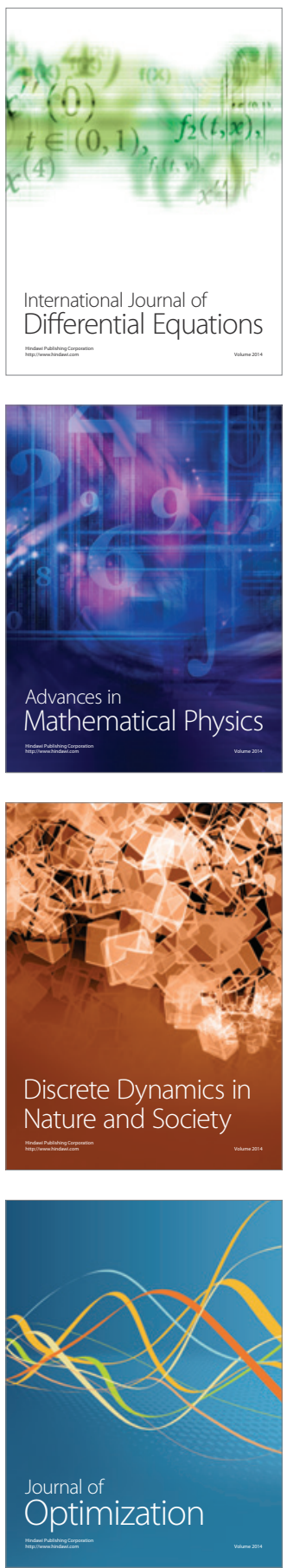